\title{
Predicting User Acceptance and Continuance Behaviour Towards Location-based Services: The Moderating Effect of Facilitating Conditions on Behavioural Intention and Actual Use
}

\author{
Mohammad Alamgir Hossain \\ RMIT University \\ mahripon@yahoo.com
}

\author{
Mohammad Imran Hasan \\ Latrobe University \\ Caroline Chan \\ RMIT University \\ Jashim Uddin Ahmed \\ North South University \\ Bangladesh
}

\begin{abstract}
The purpose of this study is to establish and examine the significance of a consumer acceptance and continuance model for location-based services (LBSs) through the integration of perceived entertainment gratification (PEG) and perceived application quality (PAQ) with the technology acceptance model (TAM). By arguing that behavioural intention (BI) does not automatically lead to actual use (AU), we investigated the moderating effect of facilitating conditions (FC) on the relationship between BI and AU. A quantitative study was conducted in Australia and Bangladesh; data were obtained from multiple sources by systematic sampling of the distribution of questionnaires. For data analysis we applied the partial least square (PLS) method. The results indicate that, in both Australia and Bangladesh, perceived usefulness (PU), PEG and PAQ have significant influence on user attitude (UA), which has a subsequent effect on BI. Interestingly, perceived ease of use (PEoU) does not have a direct effect on UA but indirectly influences it through PU-confirming the mediating effect of PU. Further, FC has a moderating effect between BI and AU. The implications of these findings and directions for future research directions are also discussed.
\end{abstract}

Keywords: location-based service, TAM, consumer acceptance and continuance, entertainment gratification, application quality

\section{Introduction}

With the proliferation of mobile technologies-as a consequence of cost and usability-mobile devices are carried by most of the people at most of the time. As geographic location of mobile devices can be identified, location specific information can be poured-in. Consequently, people are exposed to a number of location-based services (LBSs) using mobile technologies. LBSs are defined as the "services that depend on and are enhanced by positional information of mobile device[s]" (Dhar and Varshney 2011, p.122). The Australian Mobile Telecommunications Association defined them as "any information, entertainment or social media service or application available on a mobile device that makes use of the device's geographic position" (AMTA 2010, p. 4). LBSs have been evolved after the Federal Communications Commission in the USA mandated mobile telecommunication companies to acquire precise information about the location of wireless callers who called 911. Afterwards, mobile phone operators, mobile phone companies and software firms started investing significantly in the development and integration of LBS applications that could be accessible from mobile devices (Bellavista, Kupper, and Helal 2008). Today, LBS is an unprecedented way of providing information services to the community as well as an effective means of advertising for businesses. 
The overall market of LBS is growing exponentially; the revenue generated worldwide from LBS in 2005 was around $\$ 2.6$ billion and this figure had increased to almost $\$ 10$ billion by 2010 (Steinfield 2004). The four most commonly used services of LBS are emergency services, including roadside assistance, police, fire and hospital and search and rescue missions; content-and-LBSs, including weather reports, train or bus schedules and nearest petrol stations; tracking and navigation services, such as fleet tracking and locating pets or children; and location-based advertising, including promotions and customer notifications (Steinfield 2004). Based on customer locations (or preferences) information is typically supplied by a LSP in the form of text, image, map or directions. The time saving aspects of LBS along with the convenience it offers can be very valuable to the user (Pura 2005).

A reasonable number of academic studies have been conducted that contribute empirical insights into the behavioural aspects of LBS use. Investigating the intentions of customers to adopt LBS, Zhou (2012) found that privacy, trust and perceived risk are important; whereas, in another study, the same sample suggested that other factors such as contextual offerings and ubiquitous connections are similarly important (Zhou 2015). Likewise, predicting behavioural intention (BI) of potential and experienced customers to adopt LBS, Xu and Gupta (2009) found performance expectancy, effort expectancy, personal innovativeness and privacy concerns to be significant. Assessing the effect of the value perceived by customers on BI, Pura (2005) identified conditional, monetary and convenience-related factors but found epistemic value to be likewise significant. Prior studies also investigated consumers' continuance intentions and found that perceived risk, usefulness and justice (Zhou 2013), perceived privacy and service provider interventions to manage security and information quality (often considered as system quality) are important (Chin 2012, Xu and Gupta 2009, Xu, Teo, and Tan 2005).

Intention does not necessarily always confirm the actual acceptance of a technology. Likewise, in spite of existing efforts in the industry, "LBS has not been widely adopted by users" (Zhou 2013, p. 142). In Europe it has gained only $1 \%$ in data traffic (Patel 2004) and the trend is even worse in developing countries (Dano 2003, Rainio 2003). Rather than examining user attitude and intention, ascertaining actual usage criteria is important because usage is considered 'as a surrogate measure for information systems success' (Taylor and Todd 1995, p. 144). By way of a solution to this problem, this study is based upon TAM, which "has emerged as a powerful and parsimonious way to represent the antecedents of system usage through behavioural beliefs" (Taylor and Todd 1995, p. 145). Another reason for using TAM in this research is because it better explains information-systems-related behaviour than other competitive theories including the theory of planned behaviour (TPB) (Taylor and Todd 1995, p. 148).

Our study incorporates perceived entertainment gratification (PEG) and perceived application quality (PAQ) with TAM to provide a new integrated model for consumer acceptance and continuance of LBS founded on the following rationales: (i) perceived usefulness (PU) and perceived ease of use (PEoU) are used to predict consumer acceptance of LBS applications; (ii) Most individual IT users (not including employees coerced to use IT applications as a part of their job requirements) do not expect merely utilitarian benefits but also hedonic benefits from using an application. They may accept an application out of an urgent need but decide to continuance if it is enjoyable to use (Lim and Ting 2014); (iii) Given the importance of positive initial experience for accepting an application, the overall quality of the application is crucial for its continued use. It must address a variety of needs for a variety of people with effective privacy and security features; otherwise, people will uninstall it after having the initial use for a specific occasion; (iv) Further, in order to convert 'attitude' into 'intention', an external locus of control over extraneous (e.g., technological) resources is needed (Bhattacherjee, Perols, and Sanford 2008)-leading to the investigation of the moderating effect of facilitating conditions (FC). It can be reemphasised that PU and PEoU are the variables related to 'acceptance' while PEG and PAQ are related with 'continuance' of LBS applications. These four variables are considered as the antecedents of 'attitude towards use of LBS'. This approach is used by prior studies in information systems (e.g., Lim and Ting 2014). 
Most existing studies in the LBS domain were conducted using a single sample but prior information systems (IS) studies have cautioned that the same behaviour of a given model cannot be guaranteed across cultures (Aleke, Ojiako, and Wainwright 2011, Tan et al. 2010). For instance, comparing between Hong Kong and Canada, Bailey and Caidi (2005) found that differences in cultural notions of privacy may affect the acceptance of IT-based innovations. Privacy is a serious concern in Australia but less so in Bangladesh (Hossain 2014, Hossain and Dwivedi 2014). Hossain, Dwivedi, and Piercy (2015) explained that:

TAM has been tested across technologies, persons, contexts and times and has been found as a robust model with satisfactory predictive validity; however, it is not universal in terms of culture. Generally speaking, TAM is more consistent in North America and Europe but has got some reservations in Asia.

Hence, we applied the same model in two different cultures to understand its applicability across cultures. The model is validated by survey data using PLS modelling.

The organisation of the rest of this paper is as follows. In the next section we present the theoretical background and research framework used for the current study. We then describe the empirical aspects of the study, explaining the development of measurement instruments, data collection and analyses. In the final section we interpret the results and offer concluding remarks.

\section{Theoretical Background}

To understand the acceptance behaviour of individuals in relation to technological innovations, TAM is the most accepted and applied theory (Hossain, Dwivedi, and Piercy 2015). TAM signifies two determinants of technological acceptance: PU and PEoU. These directly influence user attitudes (UA) towards the use of technology that, in turn, lead to behavioural intention (BI) that ultimately drive actual use (AU). Although initially developed to investigate computer acceptance behaviours, TAM has been applied in numerous applications including e-mail, the Internet, e-commerce, software applications, knowledge management systems and modelling grammar-just to name a few (Hossain, Dwivedi, and Piercy 2015). In spite of the mass acceptance of TAM, the model still lacks some important and essential aspects for predicting consumer behaviour specific to ubiquitous technologies, such as mobile phones (Ahmad et al. 2013). Hence, many studies have extended TAM, arguing that other contextualised factors and their correlations possess better capacity to predict the BI of consumers (Mathieson, Peacock, and Chin 2001)-still the original two constructs, PU and PEoU, have always been used.

\subsection{Perceived Usefulness}

PU, which accounts for user perceptions of the benefits of IT usage, has been consistently identified in literature as a significant variable explaining the behaviour of IT users. PU is defined as the degree to which an individual believes that using LBS will reduce the time and effort required to search and obtain results from a location-based query (adapted from Davis 1989, Xu, Teo, and Tan 2005). LBS can help an individual to find locations (positioning), train or bus schedules, and weather reports (Barnes 2003, Junglas and Watson 2008). In most cases, information provided by LBS is faster as well as more accurate, convenient and precise than that gained by other means such as (street) directories (Hurson and Gao 2009, Pura 2005). The effect of PU on UA has been validated by prior IS research. In fact, PU has emerged as one of the most significant determinants of UA as evidenced by studies in mobile services (Nysveen, Pedersen, and Thorbjørnsen 2005b) and mobile banking (Bhattacherjee 2000). Prior studies have found that, as a consequence of their various advantages, LBS applications develop positive attitudes in users (e.g., Junglas, Abraham, and Watson 2008). Therefore, we propose that:

H1. Perceived usefulness will have a positive influence on user attitude to accepting a location-based service 


\subsection{Perceived Ease of Use}

'[E]ven if an application is perceived as useful, it will only be used if it is perceived as easy to use' (Davis 1989, p.320). PEoU is defined as the degree to which a person believes that using a technology will be free from effort (Davis 1989). PEoU has been a widely-used variable in IS/IT studies, including those on the Internet (Bradley 2012, Jebeile and Reeve 2007, Seyal and Rahman 2003), m-commerce (Yang 2005), and mobile services (López-Nicolás, MolinaCastillo, and Bouwman 2008, Nysveen, Pedersen, and Thorbjørnsen 2005b). PEoU has also been identified as a significant factor for location tracking and location awareness services (Junglas and Watson 2008). Usability- and complexity-related issues, such as using complicated navigation or requiring too many inputs from users, form non-positive attitudes to using applications. Among the available LBS applications, people tend to use those that require less mental effort and time; even for customised or advanced searches, applications that complete most inputs or provide easily selectable options are more preferable to users than those that request detailed inputs. Following prior studies, our next hypothesis is as follows:

H2. Perceived ease of use will have a positive influence on user attitude to accepting a location-based service

According to TAM, PU is influenced by PEoU because, in the absence of other affecting variants, the easier a system is to use the more useful it can become. Extensive empirical evidence can be observed in IS literature that suggests that PEoU is significantly linked to user attitude, both directly and indirectly, via its impact on PU (mediating effect) (e.g., Venkatesh and Davis 2000, Jebeile and Reeve 2007). Our study examines the effects of PEoU on PU because, in the absence of other modifying influences, the less effort required to use an LBS application, the more its perceived performance will be increased. Hence:

H3. Perceived ease of use will have a positive influence on the perceived usefulness of LBSs

Bhattacherjee, Perols, and Sanford (2008) argue that the initial adoption of technology is important in justifying its acceptance; however, its ultimate success depends on its continued use, as users may initially accept it, but may discontinue using it at a later stage. In its current context, despite the numerous benefits of LBS, there are growing concerns about the success of LBS applications. Following other studies, we have used 'continuance' as a dummy for the success of LBS technology; perceived entertainment gratification (PEG) and perceived application quality (PAQ) comprise the continuance criteria for this study (Lim and Ting 2014).

\subsection{Perceived Entertainment Gratification}

PEG has received much attention in IS literature in recent time. Often referred to by other names such as hedonic/emotional benefits, intrinsic motivation or perceived enjoyment, PEG refers to the degree to which LBS applications are perceived to be fun and entertaining to use and arouse positive feelings (Lim and Ting 2014). Prior studies found that users wish to experience pleasure; they rank an online service with higher value if the user experience is entertaining and more enjoyable than dull (Nysveen, Pedersen, and Thorbjørnsen 2005a). Particularly because of improvements in technology that ensure high quality mobile devices in terms of speed, graphics and intelligence, LBS applications have become more attractive than before. The inclusion of animation, graphical views of location and sound as well as features such as 'remember a search' or 'suggest to others' offer entertainment advantages for LBS applications. Numerous empirical studies relating to IS continuance identified PEG as an influential antecedent of attitude (e.g., Huang, Lin, and Chuang 2007, Leung and Wei 2000). Therefore we assume that:

$\mathrm{H} 4$. Perceived entertainment gratification will have a positive influence on user attitude to continued use of LBSs 


\subsection{Perceived Application Quality}

In IS research, especially research related to Internet and e-commerce, system quality is a commonly-applied variable. For LBS applications, system quality is related to the application itself. Functionalities such as Internet speed, the closest telecom tower and fit to screen are beyond the scope of individual applications. In general, perceived application quality (PAQ) is associated with particular applications and refers to user perceptions regarding technical levels of performance of LBS applications for two aspects: security and privacy (Lim and Ting 2014, Akter, D'Ambra, and Ray 2013). Application security is defined as the degree to which LBS applications are perceived to provide security by protecting data and information to ensure transactions without financial loss. Similarly, privacy has always been considered an important parameter for gaining confidence in LBS applications, corresponding to the respect of LBS applications for user privacy in terms of collecting, storing, using and sharing personal information (Li 2011). As LBS applications are location specific, they can link locations to user interests or at least to generic characteristics (e.g., gender and age). Further, to some extent, user movements (e.g., to casinos, brothels or suspected terrorist hubs) and browsed services (Steinfield 2004) can be tracked or traced-back (Dhar and Varshney 2011). Prior research demonstrates that PAQ has considerable influence on web based services and LBS use (Li 2011, Zhou 2013b, Steinfield 2004). Therefore, we propose the following hypothesis:

$\mathrm{H}_{5}$. Perceived application quality will have a positive influence on user attitude to continued use of LBSs

\subsection{User Attitude, Behavioural Intention and Usage Behaviour}

Prior studies have established that UA is an essential variable explaining human behaviour or BI. Initially, theory of reasoned action and TPB authenticated a direct association between UA and BI. Later, TAM re-established BI's direct dependence on UA. Several IS studies have provided empirical evidence supporting such a relationship (Seyal and Rahman 2003). For example, Kuo and Yen (2009) found that UA is the most influential factor for BI to use $3 \mathrm{G}$ mobile value-added services, while $\mathrm{Xu}$, Oh, and Teo (2009) established the relationship between UA and BI in the context of location-based advertising and mobile services (LópezNicolás, Molina-Castillo, and Bouwman 2008, Nysveen, Pedersen, and Thorbjørnsen 2005a). Based on these theoretical lenses, this study posits that users with positive attitude towards LBS applications are more likely to intend to use them than users who possess negative attitudes. Therefore:

H6. User attitude will have a positive influence on user behavioural intention to use LBSs

In TAM, actual usage behaviour (AU) is modelled as a direct function of BI. Extensive empirical studies have validated this notion. For example, investigating in the $\mathrm{m}$-commerce field, $\mathrm{Wu}$ and Wang (2005) found BI to be a factor significantly affecting AU. Such evidence has been established in electronic services for e-consumers (Hsu and Chiu 2004) and mobile services (Kargin, Basoglu, and Daim 2008). Assuming that positive intention towards LBS applications eventually drives AU, the following hypothesis is developed:

H7. Behavioural intention will have a positive influence on user's actual usage behaviour of LBSs

\subsection{Facilitating Conditions}

Triandis (1980) claimed that positively intentioned behaviour cannot occur if FCs in the environment prevent it. He argued that FC is an objective measure, whereas other studies hold FC to be a perception-based variable (e.g. Bhattacherjee 2000, Limayem and Hirt 2003). Bhattacherjee (2000) considers FC to be an external constraint, demonstrating beliefs about availability of resources as facilitating specific behaviour. He suggested that Internet-enabled services such as e-banking will become more feasible once the required technological infrastructure becomes available and effectual. In an LBS context, FC can be defined as the degree to which technical and infrastructural resources, such as sophistication of devices 
(equipped with location abilities, e.g., smartphones) and Internet connectivity, are available for access within a reasonable response time (AMTA 2010, Bhattacherjee, Perols, and Sanford 2008). Prior IS studies (e.g., Triandis 1980) have considered FC as an important variable and direct antecedent for explaining AU, although Thompson, Higgins, and Howell (1991) could not establish such a relation for personal computer use. Later studies claim that FC should be considered a direct antecedent of BI: '[t] he absence of facilitating resources represents barriers to usage and may inhibit the formation of intention and usage' (Taylor and Todd 1995, p. 153). However, contrary to Triandis (1980), Limayem and Hirt (2003) found that the link is strong between FC and BI but not between FC and AU. Interestingly, Limayem and Hirt (2003) implied a moderating effect of FC on BI and AU but did not test it.

"Facilitating conditions moderate the link between behavioural intentions and behaviour .... Even if a person has the intention to perform a particular behaviour or habitually performs the behaviour, the behaviour may not occur when the facilitating conditions do not permit it. For example, a student who intends to use an IBCT [Internet-based communication tools] to communicate with his/her peers will not be able to do so if his or her Internet connection doesn't work." (Limayem and Hirt 2003, p. 71)

Based on previous IS research that finds mixed influences of FC on $\mathrm{BI}$ and $\mathrm{AU}$ and addressing Limayem and Hirt (2003) research agenda, this current study investigates the moderating effects of $\mathrm{FC}$ on the relationship between $\mathrm{BI}$ and $\mathrm{AU}$ as hypothesised below:

H8. The relationship between $\mathrm{BI}$ and $\mathrm{AU}$ is moderated by the level of available facilitating conditions; that is, the relationship is weaker with insufficient FC and stronger with greater FC.

A conceptualised structural model demonstrating the variables and their associated relationships considered by this study is represented diagrammatically in Figure 1.

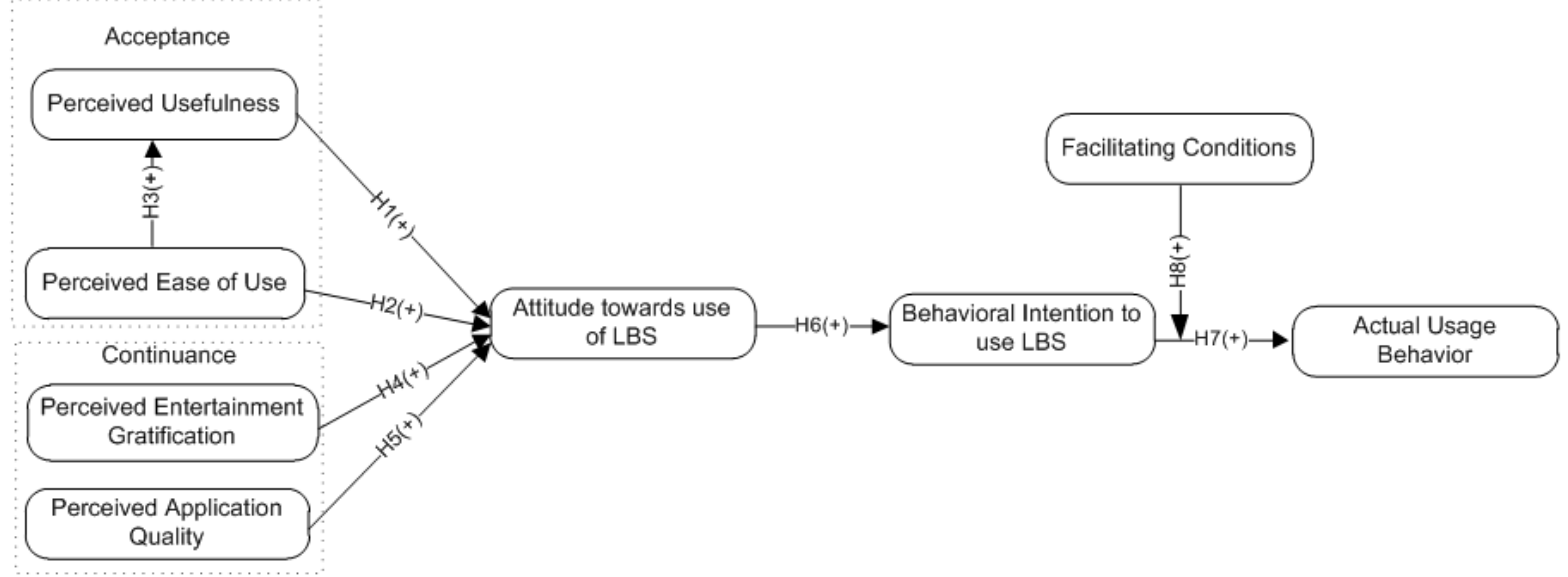

Figure 1. The Research Model

\section{Research Methods}

\subsection{Data Collection and Validation}

Data was collected from Australia and Bangladesh using 'mall-intercepted' and online surveys. The face-to-face surveys were conducted in Melbourne (Australia) and Dhaka (Bangladesh) in universities, cafés and shopping centres. These cities were chosen because they comprise both urban and suburban communities of people from different cultural, educational and professional backgrounds. Moreover, these two cities occupy respectively the top and bottom positions of the 'World's Most Liveable City' scale (The Economist 2014). Telecommunication infrastructure is one of the 30 factors considered in this ranking, where Melbourne scored the highest at 100 and Dhaka the lowest at 26.8. Therefore, considering these two cities, this study sought to discover whether availability of infrastructure really matters for LBS use. In order to 
reduce any systematic bias, the face-to-face surveys were conducted in shopping malls of different suburbs and different universities of the two cities at different days and times. Each potential respondent was approached randomly. Before answering the questionnaire, each respondent was asked if they use at least one LBS application. Moreover, an online survey was conducted using social networking groups in Facebook and Twitter. Six groups - three from each country - have been approached while only one group from each country has agreed to distribute the questionnaire among their members. Overall, the response from online survey was disappointing; only 69 responses were collected. After exhausting several attempts, a total of 219 responses were collected, but six of these were unusable because more than $10 \%$ of their values were missing. Standard demographic measures were analysed to characterise the sample (see Table 1).

\begin{tabular}{|l|l|l|l|}
\hline Categories & Distribution (\%) & \multicolumn{1}{|c|}{ Categories } & \multicolumn{1}{c|}{$\begin{array}{c}\text { Distribution } \\
\text { (\%) }\end{array}$} \\
\hline Gender & & Education & \\
\hline Male & 56 & High school & 3.51 \\
\hline Female & 44 & College & 45.96 \\
\hline Age & & Undergraduate & 38.3 \\
\hline $18-25$ & 58.16 & Postgraduate & 12.23 \\
\hline $26-33$ & 23.21 & Internet experience & \\
\hline $34-41$ & 10.58 & $<1$ year & 2.67 \\
\hline $42-49$ & 5.91 & $1-3$ years & 26.45 \\
\hline $50+$ & 2.14 & $4-5$ years & 24.67 \\
\hline & & $5+$ years & 46.21 \\
\hline
\end{tabular}

Table 1: Demographic Statistics $(n=213)$

We validated the data in three ways. First, we tested whether the responses from two samples (103 from Australia and 110 from Bangladesh) were significantly different. A Mann-Whitney $U$ test showed that there was no significant difference in the data from two independent samples. Second, we tested for non-response bias using a Kolmogorov-Smirnov (K-S) test. Using one indicator from each construct, we applied a two-sample K-S test. The non-significant $\mathrm{K}-\mathrm{S}$ test ${ }^{1}$ suggested that the sample was drawn from the same distribution. Finally, we checked the common method variance (CMV), although prior 'empirical tests of TAM have shown that it explains much of the variance in usage intention and self-reported usage' (Taylor and Todd 1995, p. 145). As a remedy for item-characteristic effects that can arise when common scale formats are used, we employed three open-ended measures to ascertain AU and Likert scale for the other measures (Sharma, Yetton, and Crawford 2009). We then applied one of the most prescribed methods-the marker variable (MV) technique (Lindell and Whitney 2001). We added an MV by deliberately including the question 'LBS are flexible to meet variety of my needs' that was theoretically unrelated to the research variables. The highest correlation ${ }^{2}$ between the MV and the latent variable (LV) was observed as BI 0.176-the maximum shared variance with other variables in the model is around $3 \%$, indicating that CMV was rather limited in our research. PLS, particularly SmartPLS (version 3.2.3, www.smartpls.de) were employed for data analysis.

\subsection{Measures}

This study consists of eight constructs. The constructs were measured using multiple-item scales drawn from pre-validated measures in IS (see Appendix A). All the measures operationalizing the constructs in the research model were considered as 'reflective' because they were interchangeable and shared one theme. Specifically, PU and PEoU were measured

\footnotetext{
${ }^{1} p$ values: $0.000,0.000,0.000,0.012,0.034,0.000,0.000,0.031$ for the indicators taken each from the derived constructs.

${ }^{2}$ The correlating values are: PU, PEoU, EG, SQ, FC, ATT, INT, USE and are equal to 0.106, 0.141, 0.155, $0.098,0.138,0.153,0.176,0.119$ respectively.
} 
by an instrument developed by Davis (1989); UA, PEG and BI were measured using a method adapted from Lim and Ting (2014) and Limayem and Hirt (2003). PAQ was ascertained using an approach taken from Akter, D'Ambra, and Ray (2013), Lim and Ting (2014), and Xu and Gupta (2009), and UB was established by adapting Bhattacherjee, Perols, and Sanford (2008). Four items that measure FC were adapted from various studies: item 1 denoted ease of access (Taylor and Todd 1995, Bhattacherjee 2000); items 2 and 3 indicated reliability and efficiency of services (Limayem and Hirt 2003); and item 4 signified the levels of assistance provided to users when they encountered difficulties (Thompson, Higgins, and Howell 1991).

\section{Results}

\subsection{Assessment of Measurement Properties}

Assessing the measurement properties of the research model following standard PLS techniques we focused on the reliability and validity of the measures representing each construct and their convergent and discriminant validities.

The first set of discriminant validity tests proves that a construct is more strongly related to its own measures than to any other construct in the model. To test this, we first calculated the average variance extracted (AVE) and compared its square root with correlations among constructs. As depicted in Table 2, all constructs met the acceptable criterion for AVE (as greater than 0.5) and thus established the reliability of the latent variables (Chin 2010). Moreover, the square root of AVE when compared to construct correlations proves that each construct is more closely related to its own measures than to other constructs (as shown on the right-hand side of Table 2). Then, the internal consistency for each block of indicators was examined by applying two measures: Cronbach's alpha provided the lower boundary of internal consistency reliability and composite reliability provided the upper boundary for the (unknown) true reliability (Hair Jr et al. 2017); both measures needed to exceed 0.7. Table 2 shows that all constructs met the threshold values.

Chin (2010) recommended that 'not only [should] each measure be strongly related to the construct it attempts to reflect, but it should not have a stronger connection with another construct' (p. 671). Therefore, we performed two tests of discriminant validity to examine how each item relates to each construct. First, the loading of each item was calculated. Referring to Igbaria, Guimaraes, and Davis (1995)'s argument, we set 0.6 as the minimum cut-off level for item reliability; following this rule, one item (ATT 2: 'I am comfortable using LBS') was discarded. It appears that for many respondents this indicator may have been indistinguishable from PEoU. Item loading checks indicated that all items effectively imitated their respective constructs (as shown by the bold values of each construct in Table 3). Later, we compared the correlations of each item to all other constructs obtained from a cross-loading matrix. Table 3 shows that no item loads higher value onto other constructs than onto the construct it represents and thus we claim that discriminant validity has been obtained at item level. 


\begin{tabular}{|c|c|c|l|l|l|l|l|l|l|l|c|}
\hline \multicolumn{3}{|c|}{ Measures of Discriminant } & \multicolumn{7}{c|}{ Inter-Correlations of the Latent Variables } \\
\hline CR & AVE & $\boldsymbol{\alpha}$ & Construct & UA & PEG & BI & FC & PEoU & PAQ & PU & AU \\
\hline 0.805 & 0.508 & 0.711 & UA & $\mathbf{0 . 7 1 3}$ & & & & & & & \\
\hline 0.851 & 0.589 & 0.768 & PEG & 0.577 & $\mathbf{0 . 7 6 7}$ & & & & & & \\
\hline 0.888 & 0.667 & 0.832 & BI & 0.526 & 0.701 & $\mathbf{0 . 8 1 6}$ & & & & & \\
\hline 0.864 & 0.614 & 0.790 & FC & 0.532 & 0.634 & 0.669 & $\mathbf{0 . 7 8 4}$ & & & & \\
\hline 0.881 & 0.650 & 0.820 & PEoU & 0.502 & 0.636 & 0.747 & 0.628 & $\mathbf{0 . 8 0 6}$ & & & \\
\hline 0.849 & 0.584 & 0.762 & PAQ & 0.577 & 0.734 & 0.696 & 0.690 & 0.695 & $\mathbf{0 . 7 6 4}$ & & \\
\hline 0.889 & 0.668 & 0.834 & PU & 0.571 & 0.618 & 0.608 & 0.599 & 0.553 & 0.572 & $\mathbf{0 . 8 1 7}$ & \\
\hline 0.860 & 0.676 & 0.759 & AU & 0.434 & 0.615 & 0.658 & 0.626 & 0.702 & 0.603 & 0.615 & $\mathbf{0 . 8 2 2}$ \\
\hline
\end{tabular}

CR - Composite reliability; AVE - Average variance extracted; $\alpha$-Chronbach's alpha; UA - User Attitude, PEG - Perceived Entertainment Gratification, BI - Behavioural Intention, FC - Facilitating Conditions, PEoU Perceived Ease of Use, PAQ - Perceived Application Quality, PU - Perceived Usefulness, AU - Actual Use; Bold diagonal values are square root of respective construct Table 2: Psychometric Properties of the Constructs

Then, we examined convergent validity to see 'how high ... each of the loadings [were] and [whether] they [were] more or less similar' (Chin 2010, p. 674). Although there was no set value prescription for the range, the observation 'the narrower the range and [the] higher the lowest loading' was taken as guide to ensure convergent validity' (Hair Jr et al. 2017). In this study we achieved relatively higher average loadings and a narrower range (from 0.674-0.892), confirming no issues with convergent validity. 


\begin{tabular}{|c|c|c|c|c|c|c|c|c|}
\hline & $\begin{array}{c}\text { User } \\
\text { Attitude }\end{array}$ & $\begin{array}{c}\text { Perceived } \\
\text { Entertainment } \\
\text { Gratification }\end{array}$ & $\begin{array}{c}\text { Behavioural } \\
\text { Intention }\end{array}$ & $\begin{array}{c}\text { Facilitating } \\
\text { Conditions }\end{array}$ & $\begin{array}{l}\text { Perceived } \\
\text { Ease of } \\
\text { Use }\end{array}$ & $\begin{array}{c}\text { Perceived } \\
\text { Application } \\
\text { Quality }\end{array}$ & $\begin{array}{c}\text { Perceived } \\
\text { Usefulness }\end{array}$ & $\begin{array}{c}\text { Actual } \\
\text { Use }\end{array}$ \\
\hline UA1 & 0.736 & 0.562 & 0.475 & 0.508 & 0.464 & 0.584 & 0.523 & 0.456 \\
\hline UA3 & 0.674 & 0.323 & 0.269 & 0.29 & 0.241 & 0.303 & 0.385 & 0.204 \\
\hline UA4 & 0.715 & 0.306 & 0.324 & 0.31 & 0.317 & 0.311 & 0.312 & 0.25 \\
\hline UA5 & 0.725 & 0.371 & 0.374 & 0.338 & 0.348 & 0.352 & 0.353 & 0.245 \\
\hline PEG1 & 0.427 & 0.738 & 0.492 & 0.517 & 0.484 & 0.597 & 0.268 & 0.368 \\
\hline PEG2 & 0.45 & 0.751 & 0.446 & 0.724 & 0.366 & 0.429 & 0.542 & 0.432 \\
\hline PEG3 & 0.367 & 0.778 & $0.55^{2}$ & 0.819 & 0.545 & 0.583 & 0.496 & 0.544 \\
\hline $\mathrm{PEG}_{4}$ & 0.506 & 0.801 & 0.65 & 0.673 & 0.559 & 0.641 & 0.573 & 0.542 \\
\hline BI1 & 0.441 & 0.575 & 0.864 & 0.569 & 0.68 & 0.577 & 0.509 & 0.59 \\
\hline BI2 & 0.455 & 0.595 & 0.851 & 0.608 & 0.604 & 0.552 & 0.533 & 0.578 \\
\hline $\mathrm{BI}_{3}$ & 0.409 & 0.537 & 0.804 & 0.494 & 0.562 & 0.574 & 0.445 & 0.483 \\
\hline $\mathrm{BI}_{4}$ & 0.41 & 0.585 & 0.74 & 0.507 & 0.589 & 0.575 & 0.494 & 0.481 \\
\hline $\mathrm{FC} 1$ & 0.396 & 0.595 & 0.582 & 0.809 & 0.547 & 0.56 & 0.458 & 0.518 \\
\hline $\mathrm{FC} 2$ & 0.473 & 0.656 & 0.507 & 0.779 & 0.491 & 0.581 & 0.389 & 0.458 \\
\hline $\mathrm{FC}_{3}$ & 0.45 & 0.751 & 0.446 & 0.724 & 0.366 & 0.429 & 0.542 & 0.432 \\
\hline $\mathrm{FC}_{4}$ & 0.367 & 0.778 & 0.552 & 0.819 & 0.545 & 0.583 & 0.496 & 0.544 \\
\hline PEoU1 & 0.327 & 0.417 & 0.508 & 0.496 & 0.745 & 0.491 & 0.441 & 0.531 \\
\hline PEoU2 & 0.41 & 0.497 & 0.557 & 0.436 & $\mathbf{0 . 8 3 1}$ & 0.599 & 0.469 & 0.559 \\
\hline $\mathrm{PEoU}_{3}$ & 0.442 & 0.589 & 0.659 & 0.577 & 0.85 & 0.582 & 0.448 & 0.612 \\
\hline $\mathrm{PEoU}_{4}$ & 0.426 & 0.529 & 0.666 & 0.515 & 0.795 & 0.56 & 0.429 & 0.559 \\
\hline PAQ1 & 0.47 & 0.486 & 0.408 & 0.479 & 0.508 & 0.709 & 0.37 & 0.39 \\
\hline PAQ2 & 0.444 & 0.654 & 0.614 & 0.568 & 0.518 & 0.777 & 0.448 & 0.46 \\
\hline PAQ3 & 0.416 & 0.575 & 0.555 & 0.518 & 0.538 & 0.764 & 0.482 & 0.528 \\
\hline PAQ4 & 0.424 & 0.527 & 0.554 & 0.542 & 0.558 & 0.804 & 0.453 & 0.463 \\
\hline PU1 & 0.505 & 0.586 & 0.564 & 0.57 & 0.49 & 0.491 & 0.788 & 0.538 \\
\hline PU2 & 0.467 & 0.552 & 0.532 & 0.508 & 0.485 & 0.538 & $\mathbf{0 . 8 3 4}$ & 0.495 \\
\hline $\mathrm{PU}_{3}$ & 0.443 & 0.451 & 0.371 & 0.436 & 0.351 & 0.431 & 0.852 & 0.424 \\
\hline $\mathrm{PU}_{4}$ & 0.445 & 0.414 & 0.506 & 0.431 & 0.47 & 0.401 & 0.793 & 0.548 \\
\hline $\mathrm{AU} 1$ & 0.383 & 0.556 & 0.575 & 0.579 & 0.663 & 0.56 & 0.545 & 0.892 \\
\hline AU2 & 0.405 & 0.557 & 0.637 & 0.548 & 0.612 & 0.556 & 0.529 & 0.881 \\
\hline $\mathrm{AU}_{3}$ & 0.262 & 0.381 & 0.366 & 0.401 & 0.431 & 0.332 & 0.446 & 0.678 \\
\hline
\end{tabular}

Table 3: Outer Model Loadings and Cross-leadings

\subsection{Assessment of the Structural Model}

For the purpose of assessing the structural model, the direction of path coefficient ( $\beta$ value) and the magnitude of $t$-statistics were checked. The results are illustrated in Figure 2 and summarised in Table 4. The results found that all our proposed hypotheses were supported except H2. As a criterion of the predictive power of the model we calculated the coefficient of determination $\left(\mathrm{R}^{2}\right)$ values of the endogenous constructs (Henseler, Ringle, and Sinkovics 2009). Our model explains $43.10 \%$ of $\mathrm{AU}, 27.70 \%$ of $\mathrm{BI}$ and $43.80 \%$ of $\mathrm{UA}\left(\mathrm{R}^{2}\right.$ values of 0.67 , 0.33, and 0.19 are considered as substantial, moderate, and weak, respectively; Henseler, 
Ringle, and Sinkovics 2009, p. 303). Moreover, we examined the effect size ${ }^{3}\left(f^{2}\right)$. The addition of two variables (namely, PAQ and PEG) into the TAM resulted in the effect size $\left(f^{2}\right)$ of 0.105 which is almost 'medium' ( $\mathrm{f}^{2}$ of $0.02,0.15$, and 0.35 represent small, medium, and large effect, Chin 2010). Therefore, it can be suggested that the model developed in this study has better explanatory power than traditional TAM for explaining LBS use.

\begin{tabular}{|c|c|c|c|c|c|}
\hline & $\begin{array}{l}\text { Path } \\
\text { coefficient }\end{array}$ & $\begin{array}{l}\text { Standard } \\
\text { Deviation } \\
\end{array}$ & $\begin{array}{c}t \\
\text { statistics } \\
\end{array}$ & $\begin{array}{c}p \\
\text { Values }\end{array}$ & $\begin{array}{c}\text { Supported } \\
?\end{array}$ \\
\hline $\begin{array}{l}\text { H1: Perceived Usefulness } \rightarrow \text { User } \\
\text { Attitude }\end{array}$ & $0.289^{* * *}$ & 0.074 & 3.927 & 0.000 & Yes \\
\hline $\begin{array}{l}\text { H2: Perceived Ease of Use } \rightarrow \text { User } \\
\text { Attitude }\end{array}$ & $0.057^{\mathrm{ns}}$ & 0.091 & 0.629 & 0.529 & No \\
\hline $\begin{array}{l}\text { H3: Perceived Ease of Use } \rightarrow \\
\text { Perceived usefulness }\end{array}$ & $0.558^{* * *}$ & 0.059 & $9 \cdot 518$ & 0.000 & Yes \\
\hline $\begin{array}{l}\text { H4: Perceived Entertainment } \\
\text { Gratification } \rightarrow \text { User Attitude }\end{array}$ & $0.194^{*}$ & 0.086 & 2.315 & 0.021 & Yes \\
\hline $\begin{array}{l}\text { H5: Perceived Application Quality } \\
\rightarrow \text { User Attitude }\end{array}$ & $0.229^{* *}$ & 0.086 & 2.645 & 0.008 & Yes \\
\hline $\begin{array}{l}\text { H6: User Attitude } \rightarrow \text { Behavioural } \\
\text { Intention }\end{array}$ & $0.526^{* * *}$ & 0.06 & 8.789 & 0.000 & Yes \\
\hline $\begin{array}{l}\text { H7: Behavioural Intention } \rightarrow \\
\text { Actual Use }\end{array}$ & $0.658^{* * *}$ & 0.044 & 15.017 & 0.000 & Yes \\
\hline
\end{tabular}

Significance level ${ }^{*} p<0.05,{ }^{* *} p<0.01,{ }^{* * *} p<0.001$

Table 4: Structural Properties of the Constructs

\subsection{Assessing the Mediating Effect}

The results show that PEoU does not affect BI but has an indirect effect through PU. This indicates the potential mediating role of PU that is strongly supported by the literature.(e.g., Venkatesh and Davis 2000). To substantiate this assertion we performed further analysis. Mediation can be assessed by examining the relationship of the indirect link via the potential mediating variable - path $a$ from the predictor (PEoU) to the mediator (PU) and path $b$ from the mediator to the endogenous variable (UA), and the direct link between predictor and endogenous variable (UA; path $c$ ) (Eberl 2010). For a mediation effect to be proven, the $z$ value 4 has to be significant. In our case, $z$ equals 3.593 which is significant at $\mathrm{p}<0.005$, but $b$ value is insignificant and thus the full mediation effect is accepted. Further, we calculated the size of the effect 5 establishing that $73.88 \%$ of the total effect of PEoU on UA is explained by PU.

\subsection{Assessing the Moderating Effect}

To examine the moderating effect of FC on the relationship between $\mathrm{BI}$ and AU, we used the PLS product-indicator approach. This process proposes building product terms between the indicators of the exogenous variable (i.e., BI) and the indicators of the latent moderator variable (i.e., FC). Consequently, the interaction term consists of 16 items (4 BI x 4 FC) (see Henseler and Fassott 2010 for detail). To analyse the moderating effect, we estimated the influence of BI and FC as well as the interaction term on UA. To claim a moderation effect, the

$3 f^{2}=\frac{R_{\text {included }}^{2}-R_{\text {excluded }}^{2}}{1-R_{\text {included }}^{2}}$

$4 Z=\frac{a * b}{\sqrt{b^{2} s \cdot e_{\cdot a}^{2}+a^{2} s \cdot e_{b}^{2}+s \cdot e_{a}^{2} s \cdot e_{b}^{2}}}$ Where $\mathrm{a}, \mathrm{b}, \mathrm{c}=$ path coefficient from PEoU to PU, PU to UA and

PEoU to attitude respectively, s.e. $=$ standard error

$5 V A F=\frac{a * b}{(a * b)+c}$ 
path coefficient between the interaction term and UA has to be significant (regardless of the values of the other two relations), which was true in our case $(\beta=0.537, S D=0.245, t=2.192$, $\left.R^{2}=0.502\right)$ and significant at $p<0.05$. To assess the strength of the moderating effect, the literature suggests using effect size $\left(f^{2}\right) .{ }^{6}$ The effect size is 'moderate' when it has an $\mathrm{f}^{2}$ value of 0.15. Thus, we confirm that FCs do moderate the relationship between BI and AU, affirming hypothesis H8.

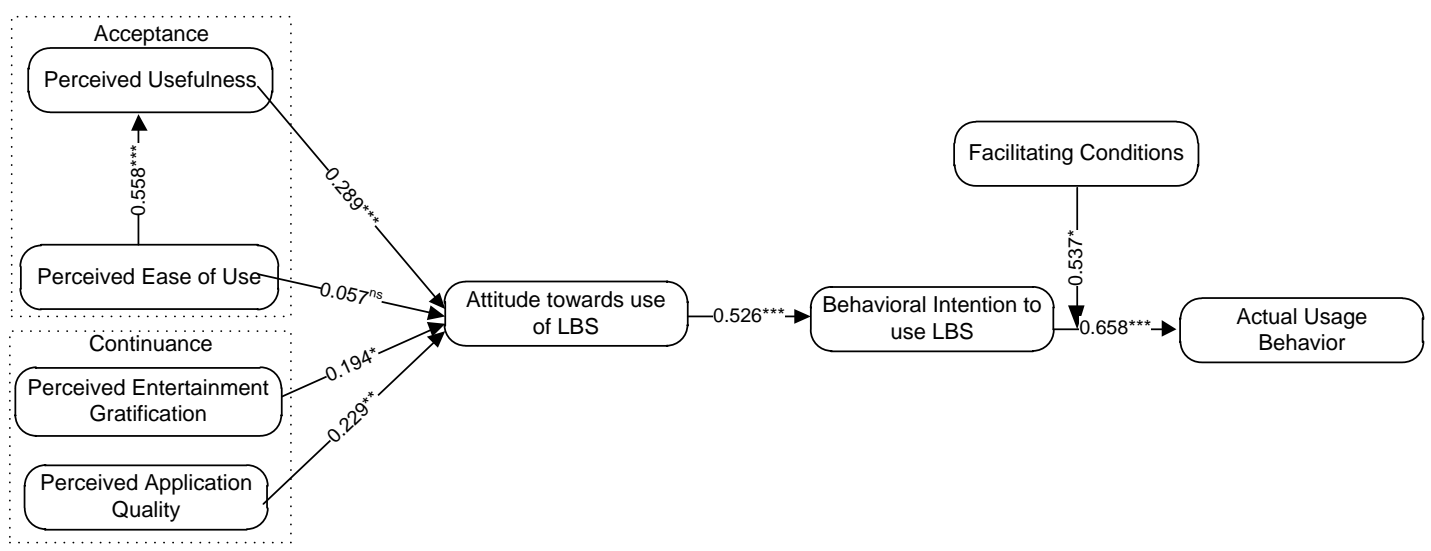

Significance level ${ }^{*} p<0.05,{ }^{* *} p<0.005,{ }^{* * *} p<0.001$; the values are $\beta$ and parenthesis are $t$-values Figure 2. The Structural Evaluation of the Model

\subsection{Multi-group Analysis}

To check whether respondent perceptions were different based on their culture (we used geographical location as a dummy), we performed multi-group analysis, which reveals significantly different group-specific PLS-SEM results for the relationships in the structural model. We compare PLS-SEM results of LBS users from Australia (Group 1) to those from Bangladesh (Group 2). Henseler, Ringle, and Sarstedt (2016) suggested that it is important to assess the measurement invariance of composite model (MICOM) in variance-based SEM using PLS. In our assessment, we apply the three steps recommended in MICOM procedure. First, in order to establish configural invariance, we ensure that the following three aspects are identical for both groups: setup of the measurement models and the structural model; data treatment for the model estimation using the full set of data and each group of data; and algorithm settings for all model estimations. In the second step, we assess the compositional invariance using the SmartPLS 3 software (Ringle et al., 2015). In principle, compositional invariance requires that $c$ equals one. A permutation test reveals if the correlation $c$ is significantly different from one or not. Table 5 shows the results of 5,000 permutations. The permutation test substantiates that the $c$ values are close to 1 and none of them are significantly different from one. We therefore conclude that compositional invariance has been established for all composites in our model. Finally, in Step 3, we assess the constructs' equality of mean values and variances across groups. The permutation test results show that the mean value and the variance of a composite in Group 1 do not significantly differ from the results in Group 2, thus full measurement invariance is established.

\footnotetext{
$6 f^{2}=\frac{R_{\text {model with moderator }}^{2}-R_{\text {model without moderator }}^{2}}{1-R_{\text {model with moderator }}^{2}}$
} 


\begin{tabular}{|c|c|c|c|}
\hline Composite & c value $(=1)$ & $\begin{array}{c}95 \% \\
\text { confidence } \\
\text { interval }\end{array}$ & $\begin{array}{c}\text { Composite } \\
\text { invariance? }\end{array}$ \\
\hline PU & 0.999 & [0.996; 1.000] & Yes \\
\hline PEoU & 0.998 & [0.996; 1.000] & Yes \\
\hline PEG & 0.995 & {$[0.988 ; 1.000]$} & Yes \\
\hline PAQ & 0.996 & {$[0.987 ; 1.000]$} & Yes \\
\hline UA & 0.989 & {$[0.971 ; 1.000]$} & Yes \\
\hline $\mathrm{BI}$ & 0.999 & {$[0.997 ; 1.000]$} & Yes \\
\hline AU & 0.997 & {$[0.988 ; 1.000]$} & Yes \\
\hline Composite & $\begin{array}{l}\text { Difference of the } \\
\text { composite's mean value } \\
(=0)\end{array}$ & $\begin{array}{c}95 \% \\
\text { confidence } \\
\text { interval }\end{array}$ & $\begin{array}{l}\text { Equal mean } \\
\text { values? }\end{array}$ \\
\hline $\mathrm{PU}$ & -0.009 & {$[-0.367-0.375]$} & Yes \\
\hline PEoU & -0.009 & [-0.349-0.345] & Yes \\
\hline PEG & -0.012 & {$[-0.331-0.409]$} & Yes \\
\hline PAQ & -0.010 & {$[-0.334-0.355]$} & Yes \\
\hline UA & -0.008 & {$[-0.387-0.413]$} & Yes \\
\hline BI & -0.010 & {$[-0.368-0.385]$} & Yes \\
\hline $\mathrm{AU}$ & -0.008 & {$[-0.316-0.321]$} & Yes \\
\hline
\end{tabular}

Table 5. MICOM results

Now, in order to assess if the relationships are different in the two different samples, we run the multi-group analysis between Australian and Bangladeshi users. We applied the permutation test as it has advantages statistical properties and recommended by the scholars (e.g., Hair Jr et al. 2017). The results, presented in Appendix B, reject variation in the two groups. That means, the hypothesised relationships developed in this research remain same in two different counties namely Australia and Bangladesh.

\section{Discussion}

\subsection{Discussion of the Main Effects}

The results established that, overall, customers accept LBS applications when they perceive them to be useful; however, to engage in continued use, customers expect applications to offer hedonic benefits and with good quality in terms of privacy and security features. This proposed and validated framework paves the way for application developers to conduct integrated analyses and design of LBS use, while underscoring that the provision of a useful service, although important, is not sufficient on its own; developers must address other associated factors, such as quality of apps, when designing LBS applications. Now we will discuss the results of each hypothesis along with their practical implications.

Supporting the first hypothesis our results found that PU influences attitudes to LBS use $(\beta=$ $0.289 ; t=3.927 ; p=0.000$ ). Our study suggests that consumers perceive that LBS applications will improve their performance and productivity when finding locations and other related information. In other words, customers form positive attitude towards LBS applications when these are perceived to be use-enhancing search initiatives (e.g., saving time and effort or providing current and trustworthy information). Given this scenario, LBS applications should be designed applying an extensive range of tools related to spatial technologies to provide optimised solutions to users. In this regard unbiased and current information would be important. In addition, customised services could be offered to users based on their search patterns. As a minimum, remembering past user searches could further improve the PU of LBS. Additionally, by adapting the frequently used visiting features of users and refining their searches accordingly, LBS applications could integrate user information with updated versions, helping users to save time and effort on their next visits. 
In contrast to the expectations of our second hypothesis (H2), PEoU is unable to significantly predict UAs $(\beta=0.057 ; t=0.629 ; p=0.529)$. In the context of LBS, Zhou (2012) likewise found that 'effort expectancy' does not influence usage intention. This is likely because LBS applications are not perceived as difficult to use and so PEoU does not necessarily play a role in forming (positive) attitudes. Indeed, as a consequence of unprecedented advancement in technologies (e.g., smartphones) and software, it is observed that UAs to technology are becoming positive. Even toddlers can now install and use applications (e.g., games) without asking for help from elders, a phenomenon that is relatively recent. At the same time, selfefficacy is increasing among users who use computer applications regularly; therefore, PEoU does not create any variance in attitude. Nonetheless, LBS applications should be built so as to require minimal effort for users to understand and use (Junglas and Watson 2008).

However, the significance of PEoU should not be underestimated because PEoU positively and strongly affects PU ( $\beta=0.558 ; t=9.518 ; p=0.000)$. Moreover, our mediation effect statistically proved that PEoU has an indirect effect on UA through $\mathrm{PU}(\beta=0.537, t=2.192)$. This finding is consistent with prior studies. Hossain, Dwivedi, and Piercy (2015) claimed that "an increasing number of TAM studies have found that PU has greater influence on BI than PEoU; whereas, PEoU has stronger effect through PU than as a direct effect on BI" (p. 806). Our results claim that, when other things remain constant, PU is influenced by PEoU, while UA is dependent on PU. This is implied as follows. The easier an LBS application is to use, the more users perceive it as useful and a useful application encourages users to form positive attitudes to using it. Consistent with our findings, prior IS studies recommend that PEoU is significantly and indirectly associated with UA through PU (e.g., Venkatesh and Davis 2000).

The fourth hypothesis posited that PEG would have a positive effect on attitudes to LBS use; the results revealed a significant contribution $(\beta=0.194 ; t=2.315 ; p=0.021)$ and thus $\mathrm{H}_{4}$ was confirmed. As contemporary customer expectations are no longer confined to utilitarian benefits but are also directed towards hedonic pleasures, there is a need for LBS applications to be entertaining and fun and to create enjoyment for users. Features such as light and sound effects (using Flash, for example) and the potential to share searches on social media, could be considered as ways of converting attitudes from dull to lively. Nonetheless, developers should not forget information quality at the cost of focusing on entertainment-rather, a blend of both concerns in equal measure might be useful.

Our fifth hypothesis was accepted $(\beta=0.229 ; t=2.645 ; p=0.008)$; as expected, a positive association exists between PAQ and UA. This finding emphasises that users do not consider the complexity of LBS applications as an issue and demonstrate comparatively little concern for enjoyment of the application; conversely, they are more concerned with the quality of the application itself, mostly as related to security and privacy. From a systems perspective, this is obviously an important issue that continually needs to be addressed. Therefore, application developers should take measures such as blocking third-party advertisements or affiliated sites, securing users from potential hazards and risks associated with privacy and security and informing users about privacy and security measures. Similarly, LSPs can encourage customers to sample trial versions and provide demonstrations and information on security and privacy mechanisms (e.g., through frequently asked questions and video tutorials).

Our next hypothesis (H6) predicted that UA to LBS use would have a positive influence on BI; the findings of this study confirm such a relationship ( $\beta=0.526 ; t=8.789 ; p=0.000)$. This means that consumers who possess favourable attitudes to LBS use will have a higher tendency to use it. One practical implication of this finding is that providers of LBS applications should conduct proper marketing initiatives to convince potential customers about their (positive) functionalities, assisting them to form positive attitudes. As the prior hypothesised relationships explain, disseminating useful features along with benefits related to enjoyment while demonstrating how applications are secure and privacy-enabled would instil confidence and help to form positive attitudes. Strategic emphases and resource allocation are of great importance during the development of LBS applications (Lim and Ting 2014). 


\subsection{Effect of Moderating Variables}

In an attempt to extend the existing literature on user intentions and AU that represents a critical but understudied area in IS, we investigated the effect of FCs. Our data analysis established that user's BI to use LBS applications would drive them to AU, strongly supporting hypothesis $\mathrm{H}_{7}(\beta=0.658 ; t=15.017 ; p=0.000)$. Further, the moderating effect calculations demonstrate that FC has a positive and significant moderating influence on the relationship between $\mathrm{BI}$ and $\mathrm{AU}$. This means that the relation between intention and $\mathrm{AU}$ is more prominent under the influence of FC. In other words, when FC is low, BI will have less effect on AU than when FC is high. The implications of this finding are enormous. The results suggest that for people intending to use LBS applications, factors including PU, PEoU or PEG are important but, when they decide to actually use LBS applications, FC is of greater concern-increased FC will lead to greater usage. In terms of practical implications, the supply-side stakeholders of LBS applications (e.g., mobile carriers and Internet providers) should ensure steady infrastructure that permits and encourages people to use LBS. The positive impact of FC should also alert system developers to seriously address potential barriers, including how downloads (of maps or addresses, for example) can be optimised in the presence of slow Internet speeds. Developers can also educate users. For instance, as the accuracy of LBS applications varies considerably depending on the settings chosen by the user, AMTA (2010) guidelines demonstrating the proper use of LBSs would benefit their usage. 'However, the presence of facilitating resources may not, per se, encourage usage' (Taylor and Todd 1995, p. 153) if other antecedents are missing or poorly managed.

\subsection{Implication of Cross-country Differences}

One of the major objectives of this study was to understand the cross-cultural differences in user perceptions towards LBS use. Our multi-group analysis shows that, in general, there is no significant difference between two cultural groups (Australia and Bangladesh, representing an individualistic and a collectivistic society, respectively). The main implication of this finding is that a major reservation concerning the limitation of the applicability of the TAM in Asian culture is not necessarily be true as long as contextual factors are incorporated-our model worked well across two different cultures. In other words, the TAM behaves well for a technology (e.g., Internet) which has almost same nature of applications across societies (Fusilier and Durlabhji 2005). For general/universal technologies, the market is progressively getting truly global. As mentioned earlier, anyone can (un)install mobile applications without asking help from others - this is not necessarily the case exclusively in the developed countries. The ease of installation, use, and uninstallation of an application thus deny the impact of several historically-proven variables such as self-efficacy, (general and computer) literacy, prior experience with technology, etc. on user acceptance and continuance. In fact, in some cases, people in developing countries use more mobile applications, or the extent of use of a particular application is much higher, than that in the developed countries (e.g., mobile health applications, Braw 2014, Gable 2015). As a result, the applicability of the TAM variables is getting universal. For example, in many instances, perceived complexity does not impact the adoption of several technologies in both developed and developing countries [for example, online banking in UK (Yousafzai, Foxall, and Pallister 2010) and India (Roy, Kesharwani, and Singh Bisht 2012)]. In other words, the unique socio-economic and demographic profiles of the end-users in underdeveloped communities do not actually have significant impact shaping the perceptions of the users toward the use of universal technologies. Hence, it is not recommended to make significant difference in the design of the functional and interface characteristics (from users' perspectives) of mobile applications based on the cultural or socioeconomic differences.

However, a more detailed look at the results (Appendix B) indicates some differences in perceptions-though not statistically significant ones. The three statistically supported antecedents (PU, PEG and PAQ) have stronger effects on Australian users, indicating that Australian users expect LBS applications to be useful, pleasant to use and safe in security and privacy measures. Conversely, although Bangladeshi users agree with these factors, they are 
less likely to be concerned about PEG; comparatively, Bangladeshi users rank PEoU higher than Australian users do. This finding is supported by cultural practice. The addressing of the mechanisms of physical properties is non-uniform (i.e., different throughout different parts of a city) and quite hard to comprehend in Bangladesh. Therefore, following the instructions provided by an LBS application is not always easy. Moreover, updating mechanisms for a change of address is unscientific and can sometimes complicate the uses of applications. Further, LBS applications are still at infancy stage; only a handful of services are currently available. Hence, Bangladeshi users still consider LBS applications as a privilege and as already too much of a luxury to expect entertainment, although the applications are required to be privacy- and security-enabled. On the contrary, Australian users have been enjoying almost every possible LBS application that they can imagine. They expect that LBS applications should be useful (e.g., providing built-in information as to whether a store is open or closed, along with general business hours), secure enough to transact (e.g., to order a drink from the nearest coffee parlour before arrival) and not susceptible to privacy violations-while, at the same time, the whole virtual journey should be enjoyable. Thus, one practical suggestion derived from this multi-group analysis is that LBS developers should be attentive when designing applications, especially those targeting individualistic societies.

\subsection{Theoretical Contributions}

The primary contribution of this study is its integration of variables associated with user acceptance (i.e., PU and PEoU) and continuance (i.e., PEG and PAQ) in a single model in the context of LBSs. Based on TAM, this study incorporated three context-based constructs and examined their relationships with behavioural variables, namely, attitudes, intentions and use. Predicting system use behaviours, this study provides an invitation for future research to consider acceptance and continuance factors using a single model, tested on two different cultures and found to have consistent results. In addition, the model makes a contribution to IS literature by introducing debate as to how user intention converts into AU. Finally, the demonstrated application of PLS path modelling techniques provides functional guidelines to emerging researchers about how to systematically analyse quantitative data obtained from two samples with moderating and mediating variables and multi-group analysis.

\subsection{Limitations and Future Research Direction}

Despite contributing new and valuable insights to LBS literature, this study has been faced with some limitations that may inform future research. First, we used a self-reported survey that may have resulted in self-selection bias. Although the CMV tests did not expose any concerns regarding data quality, it is still not possible to claim definitively that the data are free from self-reported bias in relation to use behaviour. Future research could use actual objective usage data from LBS providers. Second, the sample used in the survey was limited to two countries assumed to represent different cultures and such an assumption could result in limited generalisability. Future studies could investigate this model in different cultures and locate differences, which may assist in developing a generic acceptance and continuance model. Third, this study analysed the model using longitudinal data aiming to understand the behaviour of users at a specific time. Future studies could examine the model using crosssectional data. Further, the effect of control variables including age, education and previous experience using ubiquitous technologies would enhance the understanding of LBS behaviour. Fourth, prior studies have approached FCs by categorising them into technology- and resource-based constraints; we applied a single construct using indicators from both. Further research investigating their individual effects may help to better understand the effects of FCs. Finally, LBSs can be of two types: active and passive. Using 'active services' a user himself/herself actively locates his/her position (i.e., the location of the mobile device) when seeking information contextualised to that location. On the contrary, 'passive services' enable a user to be located by someone else-'once the user has consented to and initiated use of the service, identified third parties may view the location of that user's device without explicitly notifying the locatee each time their device is being located' (AMTA 2010, p. 5). Hence, passive services raise more concerns about potential privacy and security abuse. This current study 
focused only on active services, whereas future research could examine passive service-related issues and investigate this model in a different contextual setting.

\section{References}

Ahmad, Norita, Noha Tarek Amer, Faten Qutaifan, and Azza Alhilali. 2013. "Technology adoption model and a road map to successful implementation of ITIL." Journal of Enterprise Information Management 26 (5):553-576.

Akter, Shahriar, John D’Ambra, and Pradeep Ray. 2013. "Development and validation of an instrument to measure user perceived service quality of mHealth." Information \& Management 50 (4):181-195.

Aleke, Bartholomew, Udechukwu Ojiako, and David W Wainwright. 2011. "ICT adoption in developing countries: perspectives from small-scale agribusinesses." Journal of Enterprise Information Management 24 (1):68-84.

AMTA. 2010. AMTA Guidelines: Location based service providers. Australia.

Bailey, Stuart GM, and Nadia Caidi. 2005. "How much is too little? Privacy and smart cards in Hong Kong and Ontario." Journal of Information Science 31 (5):354-364.

Barnes, S, J. 2003. "Location-based services." e-Service Journal 2 (3):59-77.

Bellavista, Paolo, Axel Kupper, and Sumi Helal. 2008. "Location-based services: Back to the future." Pervasive Computing, IEEE 7 (2):85-89.

Bhattacherjee, Anol. 2000. "Acceptance of e-commerce services: the case of electronic brokerages." Systems, Man and Cybernetics, Part A: Systems and Humans, IEEE Transactions on $30(4): 411-420$.

Bhattacherjee, Anol, Johan Perols, and Clive Sanford. 2008. "Information technology continuance: A theoretic extension and empirical test." Journal of Computer Information Systems 49 (1):17-26.

Bradley, Joseph. 2012. "If We Build It They Will Come? The Technology Acceptance Model." In Information Systems Theory, 19-36. Springer.

Braw, Elisabeth. 2014. "Technology-based mobile health takes off in developing countries." The Guardian Accessed 18 January. https://www.theguardian.com/sustainablebusiness/technology-mobile-health-developing-countries.

Chin, Natalie Jun Pei. 2012. "Critical Success Factors of Location-Based Services."

Chin, Wynne W. 2010. "How to write up and report PLS analyses." In Handbook of partial least squares, 655-690. Springer.

Dano, M. 2003. "Location-based apps get wheels in Western Europe." RCR Wireless News 22 (8):4.

Davis, Fred D. 1989. "Perceived usefulness, perceived ease of use, and user acceptance of information technology." MIS Quarterly 13 (3):319-340.

Dhar, Subhankar, and Upkar Varshney. 2011. "Challenges and business models for mobile location-based services and advertising." Communications of the ACM 54 (5):121-128.

Eberl, Markus. 2010. "An application of PLS in multi-group analysis: the need for differentiated corporate-level marketing in the mobile communications industry." In Handbook of partial least squares, 487-514. Springer.

Fusilier, Marcelline, and Subhash Durlabhji. 2005. "An exploration of student internet use in India: the technology acceptance model and the theory of planned behaviour." CampusWide Information Systems 22 (4):233-246.

Gable, Eryn. 2015. "Developing Countries Lead the Way in Mobile-Health Technologies." Centre for Health market Innovations Accessed 17 January. 
http://healthmarketinnovations.org/blog/developing-countries-lead-way-mobilehealth-technologies.

Hair Jr, Joseph F, G Tomas M Hult, Christian Ringle, and Marko Sarstedt. 2017. A primer on partial least squares structural equation modeling (PLS-SEM): Sage Publications.

Henseler, Jörg, and Georg Fassott. 2010. "Testing moderating effects in PLS path models: An illustration of available procedures." In Handbook of partial least squares, 713-735. Springer.

Henseler, Jörg, Christian M Ringle, and Marko Sarstedt. 2016. "Testing measurement invariance of composites using partial least squares." International Marketing Review 33 (3):405-431.

Henseler, Jörg, Christian Ringle, and Rudolf Sinkovics. 2009. "The use of partial least squares path modeling in international marketing." Advances in International Marketing (AIM) 20:277-320.

Hossain, Mohammad Alamgir. 2014. "Exploring the perceived measures of privacy: RFID in public applications." Australasian Journal of Information Systems 18 (2).

Hossain, Mohammad Alamgir, and Yogesh K Dwivedi. 2014. "What improves citizens' privacy perceptions toward RFID technology? A cross-country investigation using mixed method approach." International Journal of Information Management 34 (6):711-719.

Hossain, Mohammad Alamgir, Yogesh K. Dwivedi, and Niall C. Piercy. 2015. "Technology Acceptance Model (TAM)." In The SAGE Encyclopedia of Quality and the Service Economy, edited by Su Mi Dahlgaard-Park, 806-807. Thousand Oaks: Sage.

Hsu, Meng-Hsiang, and Chao-Min Chiu. 2004. "Internet self-efficacy and electronic service acceptance." Decision Support Systems 38 (3):369-381.

Huang, Jen-Hung, Yu-Ru Lin, and Shu-Ting Chuang. 2007. "Elucidating user behavior of mobile learning: A perspective of the extended technology acceptance model." The Electronic Library 25 (5):585-598.

Hurson, Ali R, and Xing Gao. 2009. "Location-Based Services." Encyclopedia of information science and technology:2456-2461.

Igbaria, Magid, Tor Guimaraes, and Gordon B Davis. 1995. "Testing the determinants of microcomputer usage via a structural equation model." Journal of Management Information Systems 11 (4):87-114.

Jebeile, Sam, and Robert Reeve. 2007. "Explaining Intention to Use an Information Technology Innovation: an empirical comparison of the perceived characteristics of innovating and technology acceptance." Australasian Journal of Information Systems 15 (1): $137-152$

Junglas, Iris A, and Richard T Watson. 2008. "Location-based services." Communications of the ACM 51 (3):65-69.

Junglas, Iris, Chon Abraham, and Richard T Watson. 2008. "Task-technology fit for mobile locatable information systems." Decision Support Systems 45 (4):1046-1057.

Kargin, Banu, Nuri Basoglu, and Tugrul Daim. 2008. "Factors affecting the adoption of mobile services." International Journal of Services Sciences 2 (1):29-52.

Kuo, Ying-Feng, and Shieh-Neng Yen. 2009. "Towards an understanding of the behavioral intention to use $3 \mathrm{G}$ mobile value-added services." Computers in Human Behavior 25 (1):103-110.

Leung, Louis, and Ran Wei. 200o. "More than just talk on the move: Uses and gratifications of the cellular phone." Journalism \& Mass Communication Quarterly 77 (2):308-320. 
Li, Yuan. 2011. "Empirical studies on online information privacy concerns: literature review and an integrative framework." Communications of the Association for Information Systems 28 (1): 453-496.

Lim, Weng Marc, and Ding Hooi Ting. 2014. "Consumer acceptance and continuance of online group buying." The Journal of Computer Information Systems 54 (3):87-96.

Limayem, Moez, and Sabine Gabriele Hirt. 2003. "Force of habit and information systems usage: Theory and initial validation." Journal of the Association for Information Systems 4 (1): 65-97.

Lindell, Michael K, and David J Whitney. 2001. "Accounting for common method variance in cross-sectional research designs." Journal of Applied Psychology 86 (1):114-121.

López-Nicolás, Carolina, Francisco J Molina-Castillo, and Harry Bouwman. 2008. "An assessment of advanced mobile services acceptance: Contributions from TAM and diffusion theory models." Information \& Management 45 (6):359-364.

Mathieson, Kieran, Eileen Peacock, and Wynne W Chin. 2001. "Extending the technology acceptance model: the influence of perceived user resources." ACM SIGMIS Database 32 (3):86-112.

Nysveen, Herbjørn, Per E Pedersen, and Helge Thorbjørnsen. 2005a. "Explaining intention to use mobile chat services: moderating effects of gender." Journal of consumer Marketing 22 (5):247-256.

Nysveen, Herbjørn, Per E Pedersen, and Helge Thorbjørnsen. 2005b. "Intentions to use mobile services: Antecedents and cross-service comparisons." Journal of the Academy of Marketing Science 33 (3):330-346.

Patel, N. 2004. "Practical apps preferred over entertainment in cell phones, firm reports." Electronic News (North America) 50 (10):1.

Pura, Minna. 2005. "Linking perceived value and loyalty in location-based mobile services." Managing Service Quality 15 (6):509-538.

Rainio, A. 2003. Navi Program Report on Personal Navigation. edited by Liikennejaviestinta“ministerio“. Helsinki.

Roy, Sanjit Kumar, Ankit Kesharwani, and Shailendra Singh Bisht. 2012. "The impact of trust and perceived risk on internet banking adoption in India: An extension of technology acceptance model." International Journal of Bank Marketing 30 (4):303-322.

Seyal, Afzaal, and Mohd Noah Abd Rahman. 2003. "Student Use of the Internet: an extension of TAM in technical and vocational institutions in Brunei Darussalam." Australasian Journal of Information Systems 10 (2) :91-104.

Sharma, Rajeev, Philip Yetton, and Jeff Crawford. 2009. "Estimating the Effect of Common Method Variance: The Method-Method Pair Technique with an Illustration from TAM Research." MIS Quarterly 33(3):473-490.

Steinfield, Charles. 2004. "The development of location based services in mobile commerce." In E-Life after the Dot Com Bust, 177-197. Springer.

Tan, Khong Sin, Siong Choy Chong, Binshan Lin, and Uchenna Cyril Eze. 2010. "Internetbased ICT adoption among SMEs: demographic versus benefits, barriers, and adoption intention." Journal of Enterprise Information Management 23 (1):27-55.

Taylor, Shirley, and Peter A Todd. 1995. "Understanding information technology usage: A test of competing models." Information Systems Research 6 (2):144-176.

The-Economist. 2014. A Summary of the Liveability Ranking and Overview.

Thompson, Ronald L, Christopher A Higgins, and Jane M Howell. 1991. "Personal computing: Toward a conceptual model of utilization." MIS Quarterly 15(1):125-143. 
Triandis, Harry C. 1980. "Values, attitudes, and interpersonal behavior." Nebraska Symposium on Motivation, 1979.

Venkatesh, Viswanath, and Fred D Davis. 2000. "A theoretical extension of the technology acceptance model: four longitudinal field studies." Management Science 46 (2):186204.

Wu, Jen-Her, and Shu-Ching Wang. 2005. "What drives mobile commerce?: An empirical evaluation of the revised technology acceptance model." Information \& Management $42(5): 719-729$.

$\mathrm{Xu}$, Heng, and Sumeet Gupta. 2009. "The effects of privacy concerns and personal innovativeness on potential and experienced customers' adoption of location-based services." Electronic Markets 19 (2-3):137-149.

$\mathrm{Xu}$, Heng, Lih-Bin $\mathrm{Oh}$, and Hock-Hai Teo. 2009. "Perceived effectiveness of text vs. multimedia location-based advertising messaging." International Journal of Mobile Communications 7 (2):154-177.

$\mathrm{Xu}$, Heng, Hock-Hai Teo, and Bernard Tan. 2005. "Predicting the adoption of location-based services: the role of trust and perceived privacy risk." ICIS 2005 proceedings:71.

Yang, Kenneth CC. 2005. "Exploring factors affecting the adoption of mobile commerce in Singapore." Telematics and Informatics 22 (3):257-277.

Yousafzai, Shumaila Y, Gordon R Foxall, and John G Pallister. 2010. "Explaining internet banking behavior: theory of reasoned action, theory of planned behavior, or technology acceptance model?" Journal of Applied Social Psychology 40 (5):1172-1202.

Zhou, Tao. 2012. "Examining location-based services usage from the perspectives of unified theory of acceptance and use of technology and privacy risk." Journal of Electronic Commerce Research 13 (2):135-144.

Zhou, Tao. 2013. "Examining continuous usage of location-based services from the perspective of perceived justice." Information Systems Frontiers 15(1):141-150.

Zhou, Tao. 2015. "Understanding user adoption of location-based services from a dual perspective of enablers and inhibitors." Information Systems Frontiers 17(2):413-422.

Copyright: (C) 2017 Hossain, Hasan, Chan \& Ahmed. This is an open-access article distributed under the terms of the Creative Commons Attribution-NonCommercial 3.0 Australia License, which permits non-commercial use, distribution, and reproduction in any medium, provided the original author and AJIS are credited.

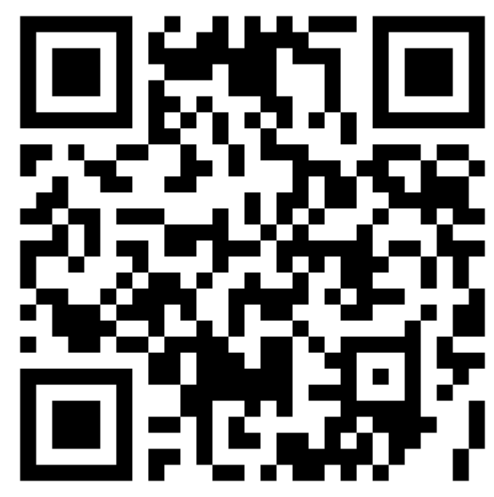




\section{Appendix A: Measurement items}

Perceived usefulness (PU) (1: Strongly disagree; 5: Strongly agree)

PU1. LBS reduce my searching time

PU2. LBS reduce my searching efforts

PU3. LBS provide me access to useful information/services

PU4. Overall, LBS are useful

Perceived Ease of Use (PEoU) (1: Strongly disagree; 5: Strongly agree)

PEoU1. My interaction with the LBS applications would be clear

PEoU2. I find the LBS applications easy to use

PEoU3. Learning to use LBS applications is easy for me

PEoU4: It would be easy for me to become skillful at using LBS applications

Perceived Entertainment Gratification (PEG) (1: Strongly disagree; 5: Strongly agree)

PEG1. LBS applications are fun to use

PEG2. I find LBS applications entertaining

PEG3. LBS applications provide me with lots of enjoyment

PEG4. LBS an exciting way to find information and services

Perceived Application Quality (PAQ) (1: Strongly disagree; 5: Strongly agree)

SQ1. LBS applications protect my personal information

SQ2. LBS applications do not share information with others

SQ3. LBS applications use sufficient security measures

SQ4. LBS applications are flexible to meet variety of needs

Facilitating Conditions (FCs) (1: Strongly disagree; 5: Strongly agree)

FC1. The LBS platform is available for most of my queries

FC2. Reliability of LBS connection is reasonable

FC3. Response time of LBS applications is reasonable

FC4. Guidance is available to me for assistance with difficulties

User Attitude (1: Strongly disagree; 5: Strongly agree)

Att1. I feel happy to use LBS applications

Att2. I am comfortable using LBS applications

Att3. I like to explore through LBS applications

Att4. I like upgrade myself with the latest information/service (e.g. new restaurant) using LBS Att5. I think using LBS is a good idea

Behavioural Intention (BI) (1: Strongly disagree; 5: Strongly agree)

Int1. I intend to use LBS applications regularly

Int2. My intention is using LBS applications than use any alternative means (e.g., street directory)

Int3. I intend to increase using LBS 
Int4. I intend to recommend LBS to others

\section{Actual Use (AU)}

AU1. Number of LBS applications I use per day: o | 1-3 | 4-6 | 7-9 | Other (specify):

AU2. Number of times I currently use LBS per day: o $\mid$ 1-3 | 4-6 | 7-9 | Other (specify):

AU3: Percentage of location-based tasks that you currently process using LBS: $0 \% \mid 1-20 \%$ | 21-40\% | 41-60\% | Other (specify):

\section{Appendix B: Result of the multi-group analysis}

\begin{tabular}{|c|c|c|c|c|c|c|c|}
\hline \multirow[b]{2}{*}{ Hypotheses } & \multicolumn{3}{|c|}{ Australian sample $(n=103)$} & \multicolumn{3}{|c|}{$\begin{array}{c}\text { Bangladeshi sample } \\
(\mathrm{n}=110)\end{array}$} & \multirow[b]{2}{*}{$\begin{array}{c}\text { Permutation } \\
p \text {-Values }\end{array}$} \\
\hline & $\begin{array}{c}\text { Path } \\
\text { coefficient }\end{array}$ & SD & $t$-Values & $\begin{array}{c}\text { Path } \\
\text { coefficient }\end{array}$ & SD & $t$-Values & \\
\hline $\mathrm{PU} \rightarrow \mathrm{UA}$ & $0.318^{* * *}$ & 0.090 & 3.529 & $0.238^{*}$ & 0.120 & 1.989 & $0.827^{\text {ns }}$ \\
\hline $\begin{array}{l}\mathrm{PEoU} \rightarrow \\
\mathrm{UA}\end{array}$ & $0.01^{\mathrm{ns}}$ & 0.121 & 0.080 & $0.104^{\mathrm{ns}}$ & 0.131 & 0.792 & $0.899^{\mathrm{ns}}$ \\
\hline $\begin{array}{l}\text { PEoU } \rightarrow \\
\text { PU }\end{array}$ & $0.456^{* * *}$ & 0.094 & 4.860 & $0.616^{* * *}$ & 0.750 & 8.201 & $0.106^{\mathrm{ns}}$ \\
\hline $\mathrm{PEG} \rightarrow \mathrm{UA}$ & $0.264^{*}$ & 0.107 & 2.475 & $0.046^{\mathrm{ns}}$ & 0.140 & 0.328 & $0.407^{\text {ns }}$ \\
\hline $\mathrm{PAQ} \rightarrow \mathrm{UA}$ & $0.203^{*}$ & 0.102 & 2.183 & $0.281^{*}$ & 0.142 & 1.978 & $0.912^{\text {ns }}$ \\
\hline $\mathrm{UA} \rightarrow \mathrm{BI}$ & $0.503^{* * *}$ & 0.083 & 6.090 & $0.475^{* * *}$ & 0.083 & 5.687 & $0.75^{\mathrm{ns}}$ \\
\hline $\mathrm{BI} \rightarrow \mathrm{AU}$ & $0.561^{* * *}$ & 0.070 & 8.071 & $0.704^{* * *}$ & 0.058 & 12.081 & $0.248^{\mathrm{ns}}$ \\
\hline
\end{tabular}

Note. SD, Standard Deviation; Significance level ${ }^{*} p<0.05,{ }^{* *} p<0.01,{ }^{* * *} p<0.001 ; n s$, not-significant 